\title{
Glycogenesis Induction
}

National Cancer Institute

\section{Source}

National Cancer Institute. Glycogenesis Induction. NCI Thesaurus. Code C40637.

Glycogenesis Induction consists of initiation of activities of biologic molecules or complexes involved in the formation of glycogen from glucose. 